BLADDER CANCER

\section{Better prediction of lymph node metastasis}

A new 51-gene signature termed KNN51 outperforms two previously developed sets in predicting lymph node metastasis in patients with muscle-invasive bladder cancer (MIBC).

Presence of lymph node metastasis puts patients with MIBC at a high risk of disease recurrence and these patients would benefit from neoadjuvant chemotherapy. However, current clinical staging is not accurate enough to capture all patients with high-risk disease and new staging tools are required to enable improved treatment planning.

In a new study published in The Journal of Urology, Roland Seiler and colleagues investigated whole-transcriptome expression profiles of radical cystectomy specimens from 199 patients with MIBC. Around $36 \%$ of these patients had lymph node metastases and around 50\% experienced disease recurrence. The team analysed total
RNA expression of the samples and included non-protein-coding transcripts, as these transcripts can have important regulatory functions in gene expression and translation.

From the discovery set of 133 tumour samples the team generated the KNN51 signature, which includes 15 unannotated and nine non-protein-coding features. The validation set comprised samples from 66 patients. The performance of two previously reported signatures, RF15 and LN20, was analysed in this set in addition to KNN51. The difference in scores between patients with lymph node metastases and those without was only significant for KNN51 $(P<0.001)$. The area under the receiver operating characteristic curve value (AUC) was highest for KNN51, followed by RF15 and LN20 (0.82, 0.62 and 0.46 , respectively).

The authors highlight that the KNN51 signature will need to be validated in samples from transurethral resection of the bladder tumour to enable its use in early patient management.

Clemens Thoma

ORIGINAL ARTICLE Seiler, R. et al. Prediction of lymph node metastasis in bladder cancer patients using whole transcriptome gene expression signatures. J. Urol. http://dx.doi.org/10.1016/j.juro.2016.04.061 (2016) 